\title{
Anemia and perioperative mortality in non- cardiac surgery patients: a secondary analysis based on a single-center retrospective study
}

Xueying Luo ${ }^{1 \dagger}$, Feng $\mathrm{Li}^{2 \dagger}$, Haofei Hu${ }^{3 \dagger}$, Baoer Liu ${ }^{4}$, Sujing Zheng ${ }^{5}$, Liping Yang ${ }^{4}$, Rui Gao ${ }^{2}, \mathrm{Ya} \mathrm{Li}^{6}$, Rao Xi ${ }^{7}$ and Jinsong $\mathrm{He}^{8^{*}}$

\begin{abstract}
Background: Evidence regarding the relationship between anemia and perioperative prognosis is controversial. The study was conducted to highlight the specific relationship between anemia and perioperative mortality in noncardiac surgery patients over 18 years of age.

Methods: This study was a retrospective analysis of the electronic medical records of 90,784 patients at the Singapore General Hospital from January 1, 2012 to October 31, 2016. Multivariate regression, propensity score analysis, doubly robust estimation, and an inverse probability-weighting model was used to ensure the robustness of our findings.

Results: We identified 85,989 patients, of whom75, 163 had none or mild anemia (Hemoglobin>90g/L) and 10,826 had moderate or severe anemia (Hemoglobin $\leq 90 \mathrm{~g} / \mathrm{L}$ ). 8,857 patients in each study exposure group had similar propensity scores and were included in the analyses. In the doubly robust model, postoperative 30-day mortality rate was increased by $0.51 \%(n=219)$ in moderate or severe anemia group (Odds Ratio, 1.510; 95\% Confidence Interval (CI), 1.049 to 2.174$)$ compared with none or mild anemia group ( $2.47 \%$ vs.1.22\%, $P<0.001)$. Moderate or severe anemia was also associated with increased postoperative blood transfusion rates (OR, 5.608; 95\% Cl, 4.026 to $7.811, P<0.001)$. There was no statistical difference in Intensive Care Unit (ICU) admission rate among different anemia groups within 30 days after surgery $(P=0.104)$.

Discussion: In patients undergoing non-cardiac surgery over 18 years old, moderate or severe preoperative anemia would increase the occurrence of postoperative blood transfusion and the risk of death, rather than ICU admission within 30 days after surgery.
\end{abstract}

Keywords: anemia, postoperative 30day mortality, non-cardiac surgery, ICU admission, postoperative transfusion, perioperative prognosis

\footnotetext{
* Correspondence: hissums1105@126.com

${ }^{+}$Xueying Luo, Feng Li and Haofei Hu contributed equally to this work.

${ }^{8}$ Department of Breast thyroid surgery, Shenzhen Breast Cancer Research and

Treatment Research Center, Peking University Shenzhen Hospital, 1120

Lianhua Road, Futian District, Shenzhen 518000, Guangdong, China

Full list of author information is available at the end of the article
}

(C) The Author(s). 2020 Open Access This article is licensed under a Creative Commons Attribution 4.0 International License, which permits use, sharing, adaptation, distribution and reproduction in any medium or format, as long as you give appropriate credit to the original author(s) and the source, provide a link to the Creative Commons licence, and indicate if changes were made. The images or other third party material in this article are included in the article's Creative Commons licence, unless indicated otherwise in a credit line to the material. If material is not included in the article's Creative Commons licence and your intended use is not permitted by statutory regulation or exceeds the permitted use, you will need to obtain permission directly from the copyright holder. To view a copy of this licence, visit http://creativecommons.org/licenses/by/4.0/ The Creative Commons Public Domain Dedication waiver (http://creativecommons.org/publicdomain/zero/1.0/) applies to the data made available in this article, unless otherwise stated in a credit line to the data. 


\section{Background}

Preoperative anemia affects $30-40 \%$ of patients undergoing major surgery and is an independent risk factor for postoperative complications and long-term mortality [1]. However, there is controversy of the relationship between anemia and perioperative prognosis, such as postoperative 30day mortality. It has been reported that the relationship between them is no statistically significant in patients undergoing rectal cancer surgery [2], cardiac surgery [3], hepatectomy [4], single-level lumbar surgery [4]. And conversely, some studies pointing out that anemia is an important predictor of 30day mortality in the patients undergoing, cardiovascular [5-7], spine tumors [8], major abdominal [9], joint arthroplasty [10], gastrointestinal surgery [11], vascular surgery [12], and thyroidectomy [13]. Little is known about the effects of anemia in the perioperative prognosis in non-cardiac surgery patients over 18 years of age, with two related studies involving children [14] and the elderly [15]. Our study aimed to investigate the relationship between different anemia status and perioperative prognosis in noncardiac surgery adult patients.

\section{Methods}

\section{Study design and setting}

This study was a secondary analysis based on a single-center retrospective study, that had been conducted a single-center retrospective study from January 1, 2012 to October 31, 2016 at the Singapore General Hospital. In the present study, it was performed to address the relationship between anemia status and perioperative prognosis. The target independent variable is anemia status obtained at baseline.

\section{Participants and Procedures}

Patients who underwent cardiac surgery, burn-related surgery, neurosurgery, and transplantation were excluded due to their categorically higher mortality rate and blood transfusion requirement, based on the original research. A total of 90785 surgical patients were recruited and selected for the study. Only surgical patients, over 18 years of age, with complete anemia data can qualified for inclusion in the study.

Covariates included in this study were specified a priori as potential confounders on the relationship of anemia and perioperative prognosis in patients, based on clinical experience and previous studies. The data collected during the preoperative anesthetic assessment visit included age, gender, race, preoperative estimated glomerular filtration rate (eGFR), presence of cerebrovascular accidents (CVA), diabetes mellitus (DM),ischemic heart disease (IHD), congestive heart failure (CHF),red cell distribution (RDW), priority of surgery, anesthesia type, surgical risk, preoperative blood transfusion with in 30days, intraoperative blood transfusion data, the
Revised Cardiac Risk Index (RCRI) score, the ASA status. Preoperative laboratory results including renal group (including eGFR) and full blood count (including hemoglobin concentration and RDW) were taken as the latest blood results within 90 days before surgery, and up to the day of surgery. RDW is the coefficient of variation (percentage) between the red blood cell volume and the normal reference range of RDW, ranging from $10.9 \%$ to $15.7 \%$. Levels $>15.7 \%$ were defined as high RDW. The severity of anemia was defined by WHO's gender-based classification of hemoglobin concentration. Mild anemia was defined as hemoglobin concentration of $11-12.9 \mathrm{~g} / \mathrm{dL}$ in males and $11-11.9 \mathrm{~g} / \mathrm{dL}$ in females; moderate anemia was defined for both genders to be hemoglobin concentration between $8-10.9 \mathrm{~g} / \mathrm{dL}$ and severe anemia defined as hemoglobin concentration $<8.0 \mathrm{~g} /$ dL. Priority of surgery (emergency or elective) and surgical risk classification were based on the 2014 European Society of Cardiology (ESC) and the European Society of Anaesthesiology (ESA) guidelines [16, 17]. American Society of Anesthesiologists-Physical Status (ASA-PS) follows that of the ASA-PS definitions [17].

The patients were followed up for 30 days after their index operation to identify all ICU admissions (stay time $>24$ hours), blood transfusion and mortality. Mortality data (the primary outcome) were synchronized with the National Electronic Health Records, ensuring a near complete follow-up [18]. The need for ICU stay (>24 hours) during surgical admission may serve as a surrogate marker for major postoperative complications.

\section{Dataset}

We downloaded the raw data for free from the DATADRYAD database (www.datadryad.org). Since Diana Xin Hui Chan et al. transferred the ownership of the original data to the DATADRYAD website, we were able to use this data for secondary data analysis based on different scientific assumptions (Dryad data package: Chan, Diana Xin Hui et al. (2018), Data from: Development of the Combined Assessment of Risk Encountered in Surgery (CARES) surgical risk calculator for prediction of postsurgical mortality and need for intensive care unit admission risk - a single-center retrospective study, Dryad, Dataset, https://doi.org/10.5061/dryad.v142481). Since our study was based on a secondary analysis of past data and the patient's personal information in the original data was anonymous, there was no need for informed consent from the participants. The ethical approval was described in the published paper [19].

\section{Statistical analysis}

Considering the differences in baseline characteristics between the two groups of eligible participants (Table 1), propensity score matching was used to identify a cohort of 
Table 1 Baseline characteristics of participants

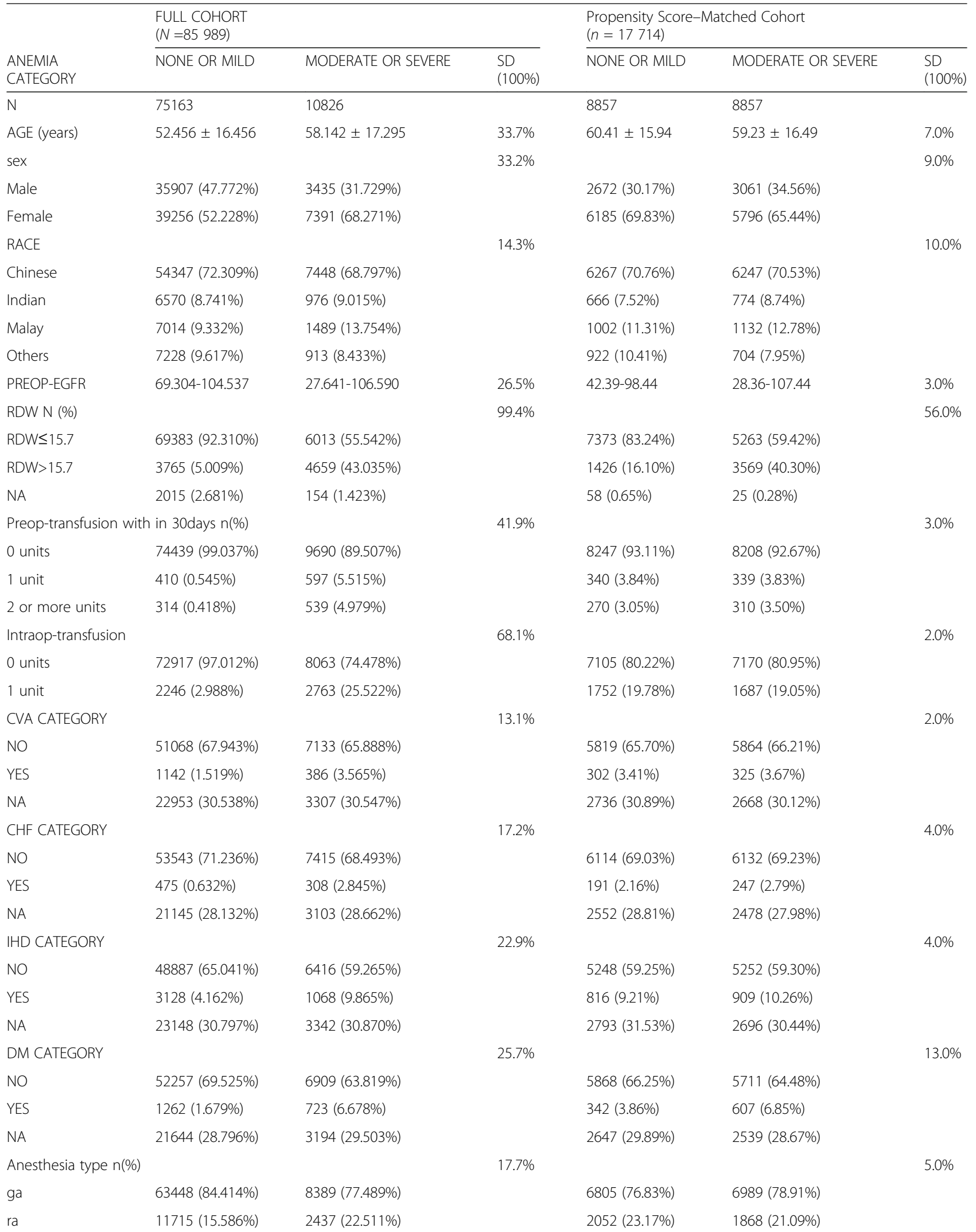


Table 1 Baseline characteristics of participants (Continued)

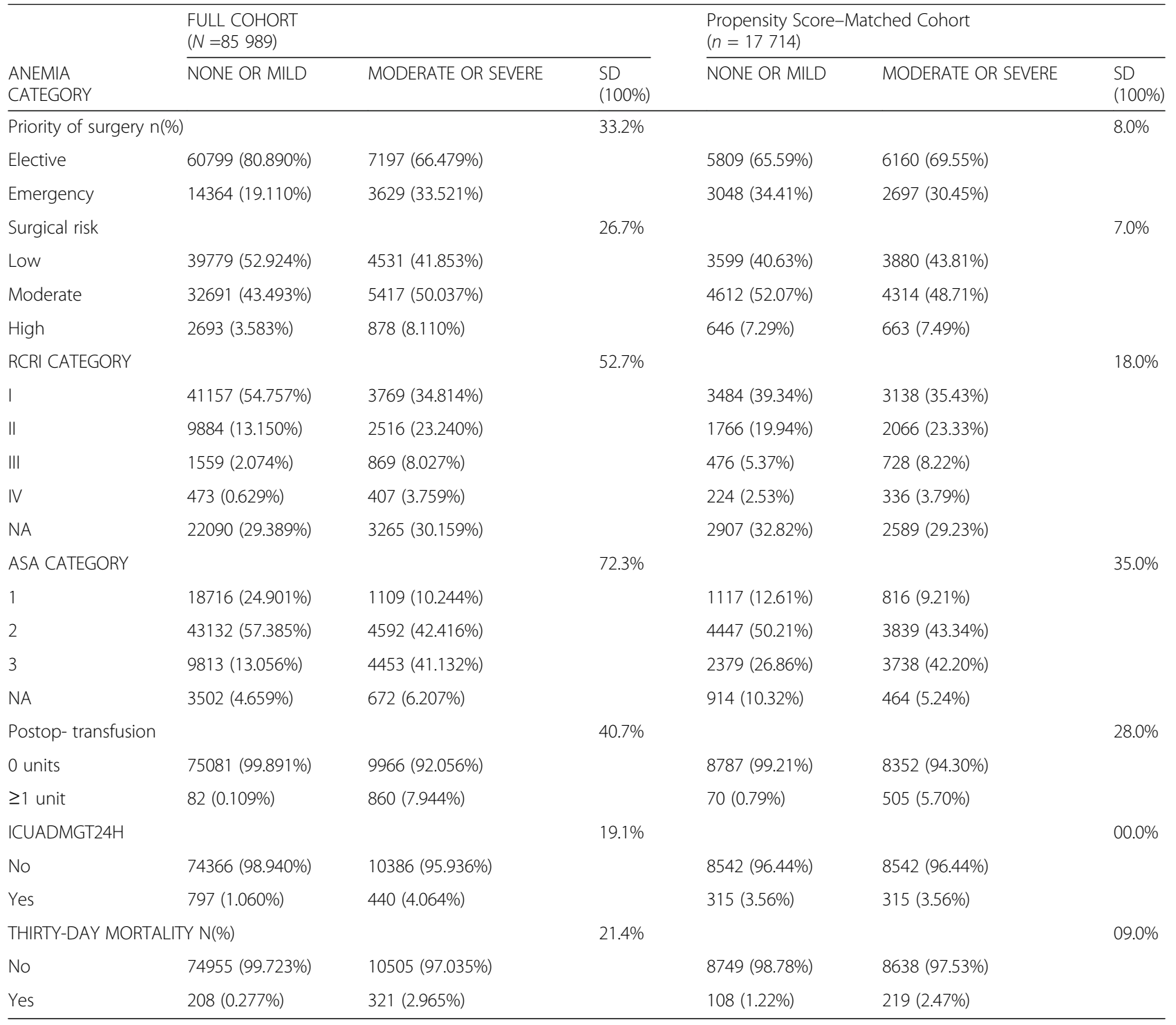

Noted: SD was calculated by Kruskal-Wallis $\mathrm{H}$ test

Abbreviations: GA general anesthesia, $R A$ regional anesthesia, PREOP-eGFR preoperative estimated glomerular filtration rate ( $\mathrm{mL} / \mathrm{min} / 1.73 \mathrm{~m} 2), R D W$ red cell distribution, NA not available, CVA cerebrovascular accidents, IHD ischemic heart disease, CHF congestive heart failure, $D M$ diabetes mellitus requiring insulin therapy; creatinine $>2.0 \mathrm{mg} / \mathrm{dl}$, Preop preoperative, Intraop intraoperative, Postop postoperative, RCRI Revised Cardiac Risk Index, ASA American Society of Anesthesiologists, ICU Intensive Care Unit, ICUADMGT24H admission to ICU for $>24$ hours

patients with similar baseline characteristics. Matching was performed with the use of a 1:1 matching protocol without replacement (greedy-matching algorithm), with a caliper width equal to 0.05 . Covariate balances before and after PS matching was assessed using standardized differences. For a given covariate, standardized differences of less than $10.0 \%$ indicate a relatively small imbalance.

The doubly robust estimation method, the combination of multivariate regression model and a propensity score model, was also applied to infer the independent associations between anemia status and patients' primary and secondary outcomes [20,21]. Using the estimated propensity scores as weights, an inverse probabilities weighting (IPW) model was used to generate a weighted cohort [22] . A logistic regression was then performed on the weighted cohort, adjusting for the variables that remained unbalanced between different anemia groups in the propensity score model.

\section{Sensitivity analysis}

We conducted a series of sensitivity analyses to evaluate the robustness of the findings of the study and how our conclusions can be affected by applying various association inference models. In the sensitivity analysis, we applied three more association inference models: a propensity score-based IPW model, a propensity score- 
based patient-matching model, and a logistic regressionbased multivariate analysis model. The calculated effect sizes and $\mathrm{p}$ values from all these models were reported and compared.

Continuous variables were expressed as mean \pm standard deviation (normal distribution) or median (interquartile range) (skewed distribution), and categorical variables were expressed in frequency or as a percentage. In the process of multivariate regression analysis, there are some confounders with partial missing data. If it is a categorical variable, the missing data would be directly treated as a new independent group; if it is a continues variable, the missing data would be replaced with an average or median value. The $\mathrm{T}$ test (normal distribution), Mann-Whitney (skewed distribution) tests and chi-square tests (categorical variables) were used to determine any statistical differences between the means and proportions of the anemia groups. All of the analyses were performed with the statistical software packages $\mathrm{R}$ (http://www.R-project.org, The $\mathrm{R}$ Foundation) and EmpowerStats (http://www.empowerstats.com, X\&Y Solutions, Inc., Boston, MA). P values less than 0.05 (two-sided) were considered statistically significant.

\section{Results}

The selection of participants

After excluding 4,037 cases with missing data of anemia status and 758 cases under 18 years of age, the study's initial cohort was recruited the initial cohort for this study was recruited( $\mathrm{N}=85$ 989;mean \pm age:53.17 \pm 16.67 years; $54.25 \%$ female ).There were $75,163(87.4 \%)$ patients with none or mild anemia, and 10,826 (12.6\%) patients with moderate or severe anemia (Fig. 1).One-to-one propensity score matching yielded 22,702 patients, with 8857 patients in each study exposure group. Patient characteristics were well balanced between exposure groups (Table 1). The standard deviation of almost all variables is less than $10 \%$, indicating that the propensity scores are perfectly matched (Figure S1).

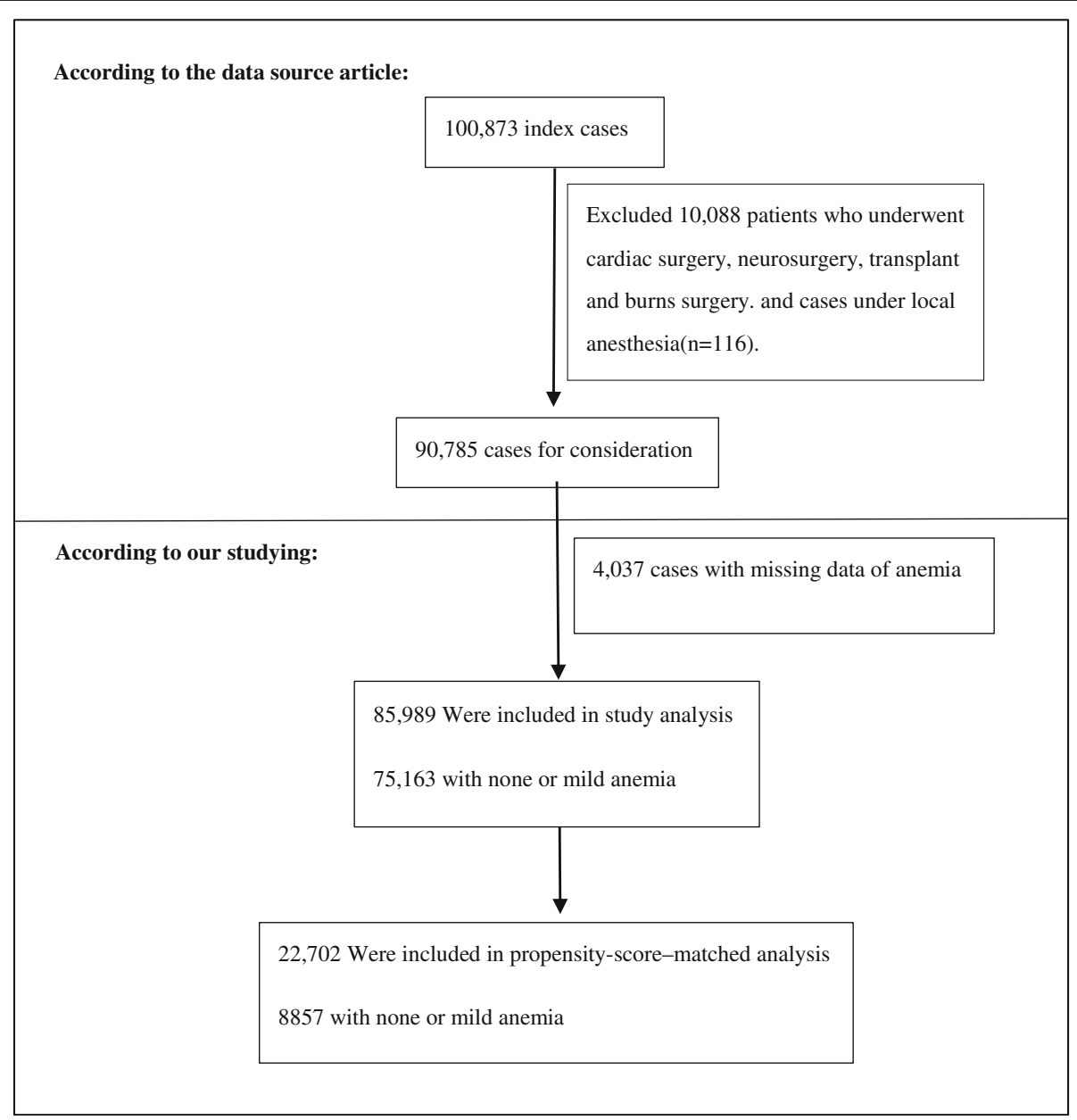

Fig. 1 Study Population 


\section{Baseline characteristics of participants}

Prior to the propensity score matching, we found that in the moderate or severe anemia group, patients were usually older, more women, more frequent preoperative and intraoperative blood transfusions, higher RDW, and a higher incidence of comorbidities, emergency surgery with higher surgical risk (based on ASA, RCRI, and surgical risk assessment). Corresponding postoperative blood transfusion times, ICU admission rates and 30-day mortality were higher. There were substantial differences between the none or mild and moderate or severe anemia groups, which highlights the need to match participants based on confounding factors. After matching at a 1: 1 ratio, we found that the included covariates were well balanced in different anemia groups. In the matching analysis, the RDW, DM, RCRI score, and ASA status are not well balanced. Therefore, we performed additional adjusted regression analysis on these variables.

\section{Outcomes}

We also showed the doubly robust estimation model, propensity score-based IPW model, and propensity score-based patient-matching model of the matched cohort in the results of multivariate analysis, and the logistic regression-based multivariate analysis model before propensity score matching (Table 2 and Table 3). In the double robust estimation model, the risk of moderate or severe anemia and postoperative blood transfusion was significantly higher than that of the group without or with mild anemia $(\mathrm{OR}=5.608$; 95\% CI, 4.026 to $7.811 ; \mathrm{P}<$ $0.001)$ and thirty-day mortality $(\mathrm{OR}=1.510,95 \% \mathrm{CI}$ : 1.049 to $2.174 ; \mathrm{P}=0.027)$. In the propensity score-based IPW model, similar relationships of moderate or severe anemia with postoperative blood transfusions (OR= 7.456, 95\% CI: 5.397 to $10.30 ; P<0.001)$ and thirty-day mortality $(\mathrm{OR}=1.996,95 \% \mathrm{CI}: 1.413$ to $2.819 ; P<0.001)$ still existed. The effect values of moderate or severe anemia were similar to those mentioned above in the propensity score-based patient-matching model (Postoperative blood transfusions: $\mathrm{OR}=8.566,95 \% \mathrm{CI}: 6.571$ to 11.17; Thirty-day mortality: $\mathrm{OR}=1.936,95 \% \mathrm{CI}: 1.530$ to 2.449), and in the logistic regression-based multivariate analysis model (Postoperative blood transfusions :OR= 7.187, 95\% CI: 5.557 to 9.296 ; Thirty-day mortality: OR= 1.917, 95\% CI: 1.531 to 2.400 ). There was no statistical difference in the admission to ICU within 30 days after surgery between different status of anemia, whether in the doubly robust estimation method ( $\mathrm{OR}=0.810,95 \%$ CI: 0.628 to $1.044 ; P=0.104)$, the propensity score-based patient-matching model (OR=0.923, 95\% CI:0.784 to 1.087; $P=0.337$, the propensity score-based IPW model (OR=0.964, 95\% CI:0.759 to $1.224 ; P=0.763$ ), and logistic regression-based multivariate analysis model $(\mathrm{OR}=0.848$, 95\% CI: 0.714 to $1.008 ; P=0.061$ ).

\section{Discussion}

This study showed that moderate or severe anemia was significantly associated with higher risks of postoperative blood transfusion and 30-day mortality in non-cardiac and non-surgery patients over 18 years of age compared to the none or mild anemia group. There was a nonsignificant relationship between different anemia status with the admission to ICU $(P=0.082)$. This finding was consistent across different statistical analyses including the doubly robust estimation method, the propensity score-based IPW model, the propensity score-based patient-matching model, and the logistic regressionbased multivariate analysis model. It revealed that the uncontrolled moderate or severe anemia before surgery would increase the occurrence of postoperative blood transfusion and the risk of death, rather than critical complications within 30 days after surgery.

Table 2 The results of univariate and multivariate analyses before propensity score matching

\begin{tabular}{|c|c|c|c|}
\hline $\begin{array}{l}\text { ANEMIA } \\
\text { CATEGORY }\end{array}$ & model i & ModEL il & ModEL ili \\
\hline \multicolumn{4}{|l|}{ Postop- transfusion } \\
\hline None or mild & Ref & Ref & Ref \\
\hline Moderate or severe & $79.01(62.936,99.194)<0.001$ & $76.924(61.075,96.885)<0.001$ & $7.187(5.557,9.296)<0.001$ \\
\hline \multicolumn{4}{|l|}{ ICUADMGT24H } \\
\hline None or mild & Ref & Ref & Ref \\
\hline Moderate or severe & $3.953(3.512,4.449)<0.001$ & $3.560(3.146,4.028)<0.001$ & $0.848(0.714,1.008) 0.061$ \\
\hline \multicolumn{4}{|l|}{ THIRTY-DAY MORTALITY } \\
\hline None or mild & Ref & Ref & Ref \\
\hline Moderate or severe & $11.011(9.238,13.126)<0.001$ & $8.395(6.989,10.084)<0.001$ & $1.917(1.531,2.400)<0.001$ \\
\hline
\end{tabular}


Table 3 The results of univariate and multivariate analyses in propensity score matched cohort

\begin{tabular}{llll}
\hline $\begin{array}{l}\text { ANEMIA } \\
\text { CATEGORY }\end{array}$ & model i* & ModEL il* & ModEL ili* $^{*}$ \\
\hline $\begin{array}{l}\text { Postop- transfusion } \\
\quad \text { None or mild }\end{array}$ & Ref & Ref & Ref \\
$\quad$ Moderate or severe & $8.566(6.571,11.17)<0.001$ & $7.456(5.397,10.30)<0.001$ & $5.608(4.026,7.811)<0.001$ \\
$\begin{array}{l}\text { ICUADMGT24H } \\
\quad \text { None or mild }\end{array}$ & Ref & Ref & Ref \\
$\quad$ Moderate or severe & $0.923(0.784,1.087) 0.337$ & $0.964(0.759,1.224) 0.763$ & $0.810(0.628,1.044) 0.104$ \\
THIRTY-DAY MORTALITY & Ref & Ref & Ref \\
$\quad \begin{array}{l}\text { None or mild } \\
\text { Moderate or severe }\end{array}$ & $1.936(1.530,2.449)<0.001$ & $1.996(1.413,2.819)<0.001$ & $1.510(1.049,2.174) 0.027$ \\
\hline
\end{tabular}

The results were expressed as odds ratio (95\%confidence interval) $P$-value

MODEL I* ( The propensity score-based patient-matching model): we adjusted for propensity score

MODEL II* (The propensity score-based IPW model):we did not adjust any covariates with the propensity score-based IPW

MODEL III*(The doubly robust estimation model): we adjusted for DM, RDW, the RCRI score, the ASA status, with the propensity score-based IPW

A consensus has been reached on the impact of anemia on long-term mortality after surgery. However, there is still considerable controversy over the effect of anemia on perioperative mortality. Many reports indicate that although anemia may increase the risk of surgical complications, it has no effect on 30-day mortality. These research groups involved patients undergoing rectal cancer surgery, cardiac surgery $[3,13,23,24]$, hepatectomy [4], singlelevel lumbar surgery [25]. Others objected to the above points, insisting that anemia is an important predictor of 30day mortality, mainly in patients undergoing cardiovascular surgery [5-7, 12]. However, studies on multidisciplinary surgical populations for non-cardiac surgery are limited. There are two related studies on this surgical population, mainly involving children [14] and the elderly [15] They have confirmed that anemia is an independent risk factor for 30-day mortality in patients of these ages, while studies of other ages are lacking. At the same time, there is currently a lack of research in different ethnic groups. Our study confirmed that there was no statistically significant difference in the effect of anemia on30-day mortality in different races over 18 years of age, highlighting the importance of controlling anemia before surgery.

This study was powered to compare anemia with perioperative prognosis. We use the doubly robust estimation method to minimize baseline differences between the groups, thus limiting the extent of treatment selection bias inherent in a retrospective study. In addition, we conducted a sensitivity analysis to confirm the reliability of the results. And this clinical database offered significant granularity in terms of demographic information, preexisting comorbidities, and risk assessment methods, which are important independent risk factors for morbidity and mortality. The prediction of the risk for postoperative ICU admission is novel and may serve as a surrogate marker for major postoperative complications.
One limitation of this study is based on a secondary analysis of published data, we can't exclude some residual and/or unmeasured confounding factors that could bias the estimated association (e.g. inflammatory markers and socioeconomic factors) and investigate the relationship between anemia with long-term outcomes. Other limitation is that although the original surgical population included most non-cardiac surgery populations, it discharged high-risk nerves, burns, etc.

\section{Conclusion}

In patients over 18 years of age undergoing non-cardiac surgery, uncontrolled moderate or severe preoperative anemia increases the incidence of postoperative blood transfusions and increases the risk of death, even if no serious complications are added within 30 days of surgery.

\section{Supplementary information}

Supplementary information accompanies this paper at https://doi.org/10. 1186/s12871-020-01024-8.

Additional file 1: Figure S1.

\section{Abbreviations}

Cl: Confidence Interval; OR: Odds Ratio; ICU: Intensive Care Unit; eGFR: estimated Glomerular Filtration Rate; CVA: Cerebrovascular accidents; CHF: Congestive Heart Failure; IHD: Ischemic Heart Disease; DM: Diabetes Mellitus; OR: Odds Ratio; Cl: Confidence Interval; CKD: Chronic Kidney Disease; CARES: Combined Assessment of Risk Encountered in Surgery; RCRI score: Revised Cardiac Risk Index Score; ASA-PS: American Society of Anesthesiologists-Physical Status; RDW: Red Cell Distribution Width; eHINTS: SingHealth-IHiS Electronic Health Information System; ESC: European Society of Cardiology; WHO: World Health Organization; GAM: Generalized Additive Model

\section{Acknowledgements}

The author is very grateful to the data providers of the study. They completed the entire study. They are (the rankings and institutions of these researchers were ranked according to the "reference [20]") Dr Hairil Rizal Abdullah (corresponding author) (Department of Anaesthesiology, Singapore 
General Hospital, Duke-NUS Medical School, Singapore), Diana Xin Hui Chan, Yilin Eileen Sim, Ruban Poopalalingam, (Department of Anaesthesiology, Singapore General Hospital, Singapore), Yiong Huak Chan (Biostatistics Unit, Yong Loo Lin School of Medicine, National University of Singapore, Singapore). The authors also thank Mr Koh Yee Jin (principal systems specialist, Department of Health Insights, Integrated Health Information Systems Pte Ltd, Singapore) and Ms Sudha Harikrishnan (Department of Anaesthesiology) for helping with data extraction process. The authors thank Professor Ong Biauw Chi (Chair ofMedical Board, Sengkang General Hospital, Singapore) for her insightful mentorship. Availability of data and materials Data can be downloaded from 'DATADRYAD' database (www.Datadryad.org).

\section{Authors' contributions}

XYL: Conceptualization; Data curation; Formal analysis; Investigation; Roles/ Writing - original draft; FL: Data curation; Formal analysis; Investigation; Software;RX and YL: Supervision; Validation; Visualization; SJZ: Supervision; Validation; Visualization; HFH: Formal analysis;Project administration;Writing review \& editing. JSH: Conceptualization; Data curation; Formal analysis;Funding acquisition; Writing - review \& editing. All authors approved the version to be published, and agree to be accountable for all aspects of the working in ensuring that questions related to the accuracy or integrity of any part of the work are appropriately investigated and resolved.

\section{Funding}

This work was funded by the Shenzhen International Cooperation Research Project (with University of Minnesota cooperation) [grant numbers GJHZ20180928115030292] and Sanming Project of Medicine in Shenzhen [grant numbers SZSM201612010],but the funders had no role in study design,data collection and analyasis,decision to publish,or preparation of the manuscript. The article-processing charges was supported by the funders.

\section{Availability of data and materials}

The data was obtained from 'DATADRYAD' database (www.Datadryad.org). This website permitted users to freely download the raw data. (Dryad data package: Development of the Combined Assessment of Risk Encountered in Surgery (CARES) surgical risk calculator for prediction of post-surgical mortality and need for intensive care unit admission risk - a single-center retrospective study, Dryad, Dataset, https://doi.org/10.5061/dryad.v142481).

\section{Ethics approval and consent to participate}

Not applicable

\section{Consent for publication}

Not applicable

(Since the study was based on a secondary analysis of past data and the patient's personal information in the original data was anonymous, there was no need for informed consent from the participants.)

\section{Competing interests}

The authors declare that they have no competing interests.

\section{Author details}

'Department of Plastic and reconstructive, Shenzhen People's Hospital, No. 1017, Dongmen North Road, Luohu District, Shenzhen ,518000, Guangdong, China. ${ }^{2}$ Department of Breast thyroid surgery, Shenzhen Breast Cancer Research and Treatment Research Center, Peking University Shenzhen Hospital, Shenzhen, China. ${ }^{3}$ Department of Breast thyroid surgery, Shenzhen Breast Cancer Research and Treatment Research Center, Peking University Shenzhen Hospital, Shenzhen, China. ${ }^{4}$ Department of Breast thyroid surgery, Shenzhen University, No. 3688 Nanhai Avenue, Nanshan District, Shenzhen 518000, Guangdong, China. ${ }^{5}$ Department of Thyroid and Breast surgery, Shenzhen Second People's Hospital, No. 3002, Sungang West Road, Futian District, Shenzhen, Shenzhen 518000, Guangdong, China. ${ }^{6}$ Department of General Medicine, Shenzhen University, No. 3002, Sungang West Road, Futian District, Shenzhen 518000, Guangdong, China. 'Department of Radiation Oncology, Faculty of Medicine, Universitatsklinikum Freiburg, Freiburg, Germany. ${ }^{8}$ Department of Breast thyroid surgery, Shenzhen Breast Cancer Research and Treatment Research Center, Peking University Shenzhen Hospital, 1120 Lianhua Road, Futian District, Shenzhen 518000, Guangdong, China.
Received: 26 January 2020 Accepted: 26 April 2020

Published online: 11 May 2020

\section{References}

1. Sim YE, et al. Prevalence of preoperative anemia, abnormal mean corpuscular volume and red cell distribution width among surgical patients in Singapore, and their influence on one year mortality. PLoS One. 2017; 12(8):e0182543.

2. Bruns ERJ, et al. The Association of Preoperative Anemia and the Postoperative Course and Oncological Outcome in Patients Undergoing Rectal Cancer Surgery: A Multicenter Snapshot Study. Dis Colon Rectum. 2019;62(7):823-31

3. Lv Z, et al. Preoperative Anemia and Postoperative Mortality in Patients with Aortic Stenosis Treated with Transcatheter Aortic Valve Implantation (TAVI): A Systematic Review and Meta-Analysis. Med Sci Monit. 2019;25:7251-7.

4. Tohme S, et al. Preoperative anemia and postoperative outcomes after hepatectomy. HPB (Oxford). 2016;18(3):255-61.

5. Miceli A, et al. Preoperative anemia increases mortality and postoperative morbidity after cardiac surgery. J Cardiothorac Surg. 2014;9:137.

6. Joshi SS, et al. Propensity-matched analysis of association between preoperative anemia and in-hospital mortality in cardiac surgical patients undergoing valvular heart surgeries. Ann Card Anaesth. 2015;18(3):373-9.

7. Padmanabhan $\mathrm{H}$, et al. Preoperative Anemia and Outcomes in Cardiovascular Surgery: Systematic Review and Meta-Analysis. Ann Thorac Surg. 2019;108(6):1840-8.

8. Hersh EH, et al. Perioperative Risk Factors for Thirty-Day Morbidity and Mortality in the Resection of Extradural Thoracic Spine Tumors. World Neurosurg. 2018;120:e950-6.

9. Abdullah HR, et al. Preoperative ANemiA among the elderly undergoing major abdominal surgery (PANAMA) study: Protocol for a single-center observational cohort study of preoperative anemia management and the impact on healthcare outcomes. Medicine (Baltimore). 2018;97(21):e10838.

10. Lu M, et al. Preoperative Anemia Independently Predicts 30-Day Complications After Aseptic and Septic Revision Total Joint Arthroplasty. J Arthroplasty. 2017;32(9s):S197-s201.

11. Lee JY, et al. Perioperative risk factors for in-hospital mortality after emergency gastrointestinal surgery. Medicine (Baltimore). 2016;95(35):e4530.

12. Gupta PK, et al. Preoperative anemia is an independent predictor of postoperative mortality and adverse cardiac events in elderly patients undergoing elective vascular operations. Ann Surg. 2013;258(6):1096-102.

13. Burton BN, et al. Association of Preoperative Anemia With 30-Day Morbidity and Mortality Among Patients With Thyroid Cancer Who Undergo Thyroidectomy. JAMA Otolaryngol Head Neck Surg. 2019;145(2):124-31.

14. Faraoni D, DiNardo JA, Goobie SM. Relationship Between Preoperative Anemia and In-Hospital Mortality in Children Undergoing Noncardiac Surgery. Anesth Analg. 2016;123(6):1582-7.

15. Wu WC, et al. Preoperative hematocrit levels and postoperative outcomes in older patients undergoing noncardiac surgery. Jama. 2007;297(22):2481-8.

16. Glance LG, et al. The Surgical Mortality Probability Model: derivation and validation of a simple risk prediction rule for noncardiac surgery. Ann Surg. 2012;255(4):696-702.

17. Kehmeier ES, Schulze VT. Cardiovascular assessment and management prior to non-cardiac surgery. Comment on the new 2014 ESC/ESA guidelines. Herz. 2015;40(8):1043-7.

18. Chan DXH, et al. Development of the Combined Assessment of Risk Encountered in Surgery (CARES) surgical risk calculator for prediction of postsurgical mortality and need for intensive care unit admission risk: a single-center retrospective study. BMJ Open. 2018;8(3):e019427.

19. McCaffrey DF, et al. A tutorial on propensity score estimation for multiple treatments using generalized boosted models. Stat Med. 2013;32(19):3388414.

20. McCaffrey DF, et al. A tutorial on propensity score estimation for multiple treatments using generalized boosted models. Stat Med. 2013; 32(19):3388-414.

21. Koch B, Vock DM, Wolfson J. Covariate selection with group lasso and doubly robust estimation of causal effects. Biometrics. 2018;74(1):8-17.

22. Cole SR, Hernan MA. Constructing inverse probability weights for marginal structural models. Am J Epidemiol. 2008;168(6):656-64.

23. Tauriainen $\mathrm{T}$, et al. The Effect of Preoperative Anemia on the Outcome After Coronary Surgery. World J Surg. 2017;41(7):1910-8. 
24. Mirhosseini SJ, Sayegh SA. Effect of preoperative anemia on short term clinical outcomes in diabetic patients after elective off-pump CABG surgery. Acta Med Iran. 2012;50(9):615-8.

25. Kim BD, et al. Preoperative anemia does not predict complications after single-level lumbar fusion: a propensity score-matched multicenter study. Spine (Phila Pa 1976). 2014;39(23):1981-9.

\section{Publisher's Note}

Springer Nature remains neutral with regard to jurisdictional claims in published maps and institutional affiliations.

Ready to submit your research? Choose BMC and benefit from:

- fast, convenient online submission

- thorough peer review by experienced researchers in your field

- rapid publication on acceptance

- support for research data, including large and complex data types

- gold Open Access which fosters wider collaboration and increased citations

- maximum visibility for your research: over $100 \mathrm{M}$ website views per year

At BMC, research is always in progress.

Learn more biomedcentral.com/submissions 